\title{
A GENERAL TURÁN EXPRESSION \\ FOR THE ZETA FUNCTION
}

\author{
H. W. Gould ${ }^{1}$ \\ (received June 30, 1962)
}

1. Introduction. In 1948 Gabor Szegö [9] gave four proofs of a remarkable inequality communicated to him by Paul Turán, who later published an original proof [10]. The Turán theorem states that if $\mathrm{P}_{n}(\mathrm{x})$ is the Legendre polynomial, then

(1.1) $\quad P_{n}^{2}(x)-P_{n+1}(x) P_{n-1}(x) \geq 0$, for $n \geq 1, \quad|x| \leq 1$, with equality holding only when $|x|=1$.

Since then many similar inequalities have been found for various special functions, particularly for the Legendre and Hermite polynomials. Reference may be had to the recent work of Danese [2] and Chatterjea [1]. Danese gives an extensive bibliography.

Not only have inequalities been found, but also explicit forms and transformations of Turán expressions have been given.

Let us define a general Turán functional operator $T$ by

$$
T_{x, a, b}(f)=T_{x} f(x)=f(x+a) f(x+b)-f(x) f(x+a+b) .
$$

The writer $[3,4,5]$ has found that this general operator enjoys a number of remarkable properties, and we might mention a few simple examples:

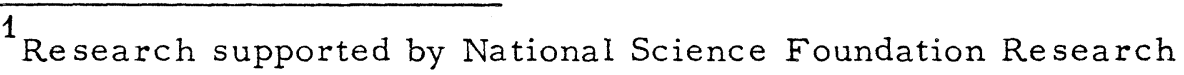
Grant G-14095.

Canad. Math. Bull. vol. 6, no. 2, September 1963 


$$
\begin{aligned}
& T(x)=a b, \\
& T(\sin x)=T(\cos x)=\sin a \sin b,
\end{aligned}
$$

$$
\begin{aligned}
& T(\sinh x)=-T(\cosh x)=\sinh a \sinh b \\
& T(\exp (c+d x))=0 \quad(c, d \text { constant })
\end{aligned}
$$

and in a problem in the American Mathematical Monthly [12] it was shown that for the Fibonacci numbers

$$
T_{n}\left(f_{n}\right)=(-1)^{n_{f}} f_{b},
$$

where $f_{1}=1=f_{2}$ and $f_{n+2}=f_{n+1}+f_{n}$.

The writer [3] found that the relation for the hyperbolic trigonometric sine and cosine can be generalized to give

$$
T_{x}\left(p u^{x+c}+q v^{x+c}\right)=-p q(u v)^{x+c}\left(u^{a}-v^{a}\right)\left(u^{b}-v^{b}\right)
$$

where $p, q, u, v, c$ are constants. This also generalizes (1.4).

In view of the elegance of the various explicit relations which may be found for the Turán operator $T$, it may be of interest to show what may be done for the Zeta function, which we shail define by

(1.6) $\quad \zeta(x)=\sum_{n=1}^{\infty} \frac{1}{n^{x}}, x>1$.

There is no difficulty in extending the results to complex $x$, so that we shall suppose $\mathrm{x}$ to be real. Our results depend on some very simpie number-theoretic calculations.

2. General Turán expression for the Zeta function.

To transform the general Turán expression

$$
T \zeta(x)=\zeta(x+a) \zeta(x+b)-\zeta(x) \zeta(x+a+b)
$$


we shall need the fact $[6$, p. 250$]$ that

$$
\zeta(\mathrm{x}) \zeta(\mathrm{x}-\mathrm{a})=\sum_{\mathrm{n}=1}^{\infty} \frac{\mathrm{a}^{(n)}}{\mathrm{n}}, \mathrm{x}>1, \mathrm{x}-\mathrm{a}>1,
$$

and where

$$
\sigma_{\mathrm{a}}(\mathrm{n})=\underset{\mathrm{d} \mid \mathrm{n}}{\sum} \mathrm{d}^{\mathrm{a}}, \quad \sigma_{0}(\mathrm{n})=\underset{\mathrm{d} \mid \mathrm{n}}{\sum} 1=\tau(\mathrm{n}) .
$$

THEOREM 1. Let $x>2$. Then

(2. 3)

$$
\begin{aligned}
& \mathrm{T}_{\mathrm{x}, 1,-1} \zeta(\mathrm{x})=\zeta(\mathrm{x}+1) \zeta(\mathrm{x}-1)-\zeta^{2}(\mathrm{x}) \\
& =\sum_{n=1}^{\infty} \frac{1}{n^{x+1}} \sum_{\substack{d \mid n \\
d<\sqrt{n}}}\left(\frac{n}{d}-d\right)^{2} .
\end{aligned}
$$

Proof. From (2.2) it is readily verified that

$$
\mathrm{T}_{\mathrm{x}, 1,-1} \zeta(\mathrm{x})=\sum_{\mathrm{n}=1}^{\infty} \frac{\sigma_{2}(\mathrm{n})-\mathrm{n} \tau(\mathrm{n})}{\mathrm{n}^{\mathrm{x}+1}},
$$

and

$$
\begin{aligned}
& \sigma_{2}(n)-n \tau(n)=\sum_{d / n}\left(d^{2}-n\right) \\
& =\Sigma\left(d^{2}-n\right)+\sum\left(d^{2}-n\right) \\
& d \mid n \quad d / n \\
& \mathrm{~d}>\sqrt{\mathrm{n}} \quad \mathrm{d}<\sqrt{\mathrm{n}} \\
& \left.=\sum_{d \mid n} \cdot\left(\frac{n}{d}\right)^{2}-n\right)+\sum_{d \mid n}\left(d^{2}-n\right) \\
& \mathrm{d}<\sqrt{\mathrm{n}} \quad \mathrm{d}<\sqrt{\mathrm{n}} \\
& =\sum_{\mathrm{d} l \mathrm{n}}\left(\frac{\mathrm{n}}{\mathrm{d}}-\mathrm{d}\right)^{2} \text {. } \\
& \mathrm{d}<\sqrt{\mathrm{n}}
\end{aligned}
$$


Since the coefficients are all positive we have the corollary that $T_{x, 1,-1} \zeta(x)>0$ for $x>2$.

THEOREM 2. Let $x>1, x+a>1, x+b>1, x+a+b>1$. Then

$$
T_{x, a, b} \zeta(x)=-\sum_{n=1}^{\infty} \frac{1}{x+a+b} \sum_{\substack{d \\ d<\sqrt{n}}}\left[\left(\frac{n}{d}\right)^{b}-d^{b}\right]\left[\left(\frac{n}{d}\right)^{a}-d^{a}\right] .
$$

Proof. Let $\mathrm{a} \geq \mathrm{b}$. Then it follows again from (2.2) that

$$
T_{x, a, b} \zeta(x)=\sum_{n=1}^{\infty} \frac{1}{n}\left[n^{b} \cdot \sigma_{a-b}(n)-\sigma_{a+b}(n)\right],
$$

and

$$
\begin{aligned}
& n^{b} \cdot \sigma_{a-b}(n)-\sigma_{a+b}(n)=\sum_{d / n} d^{a}\left[\left(\frac{n}{d}\right)^{b}-d^{b}\right] \\
& =\sum_{d \mid n} d^{a}\left[\left(\frac{n}{d}\right)^{b}-d^{b}\right]+\sum_{d \mid n} d^{a}\left[\left(\frac{n}{d}\right)^{b}-d^{b}\right] \\
& d<\sqrt{n} \\
& \text { d }>\sqrt{n} \\
& =\sum_{\substack{d \mid n \\
d<\sqrt{n}}} d^{a}\left[\left(\frac{n}{d}\right)^{b}-d^{b}\right]+\sum_{\substack{d \\
d<\sqrt[n]{n}}}^{\sum_{d}\left(\frac{n}{d}\right)^{a}\left[d^{b}-\left(\frac{n}{d}\right)^{b}\right]} \\
& =\sum_{\substack{d \\
d<\sqrt{n}}}\left[d^{a}-\left(\frac{n}{d}\right)^{a}\right]\left[\left(\frac{n}{d}\right)^{b}-d^{b}\right]
\end{aligned}
$$

from which the desired result follows. It is interesting to compare this with the relation (1.5) above. The theorem also provides a number of inequalities depending on the values of $a$ and $b$. 
It is also interesting to compare (2.4) with the corresponding result for binomial coefficients

$$
T_{x, a, b}\left(\begin{array}{l}
x \\
n
\end{array}\right)=\left(\begin{array}{c}
x+a+b \\
n
\end{array}\right) \underset{k=1}{\sum}\left(\begin{array}{c}
x \\
n-k
\end{array}\right) \frac{\left(\begin{array}{l}
a \\
k
\end{array}\right)\left(\begin{array}{l}
b \\
k
\end{array}\right)}{\left(\begin{array}{c}
x+a+b \\
k
\end{array}\right)},
$$

which is developed in [5] along with expressions for Hermite polynomials, the Bessel function, and various others. There the operator $T$ as defined by (1.2) is considered as merely one special case of the more general operator

$$
T_{x, a, b} f(x)=f(x \cup a) \cap f(x \cup b) * f(x) \cap f(x \cup a \cup b)
$$

This general operator is suggested by (1.2) if we replace + by $\cup$ and $\cdot$ by $\cap$. The abstract operations $\cup$, $\cap$ could be defined in various ways: set union and intersection, I. u.b. and g.I.b., I.c.m. and g.c.d., etc. This general operator suggested the present application to the Zeta function, and by taking * to be ordinary division instead of subtraction we shall define an operator $R$ which is related to some work of Ramanujan.

Four other operators considered in [5] and which are special cases of (2.7) are

$$
\begin{aligned}
& S(f)=f(x+a)+f(x+b)-f(x)-f(x+a+b), \\
& A(f)=f(x+a)+f(x+b)+f(x)+f(x+a+b), \\
& P(f)=f(x+a) f(x+b) f(x) f(x+a+b), \\
& R(f)=\frac{f(x+a) f(x+b)}{f(x) f(x+a+b)} .
\end{aligned}
$$

It may be of interest to point out that the two linear operators $\mathrm{S}$ and $\mathrm{A}$ may be extended in a natural way, using the Bernoulli and Euler polynomials of higher order as developed by Nörlund [7]. Indeed we could define (following Nörlund)

$$
S_{x ; a_{1}}^{n}, a_{2}, \ldots, a_{n}(f)=\left(\begin{array}{ccc}
n & & \\
i=1 & a_{i} & x, a_{i}
\end{array}\right) f(x)
$$


and

(2.13)

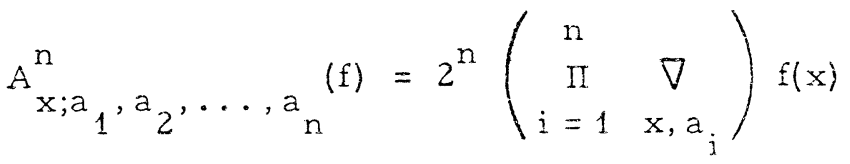

where

$$
\Delta f(x)=\frac{f(x+h)-f(x)}{h} \text { and } \underset{x, h}{x, h}(x)=\frac{f(x+h)+f(x)}{2} \text {, }
$$

which are studied by Nörlund using generalized Bernoulli and Euler polynomials of higher order; he develops inverse operators and solves functional equations involving the se operators.

The five operators T, S, A, P, R are related by various formulas developed in [5], and we wish to mention in particular the fact that

$$
P(f)=-\frac{T(f)}{T(1 / f)} \text {. }
$$

This relation suggests that having found $T(\zeta)$ it might be of values to determine $T(1 / \zeta)$ and $P(\zeta)$.

Ramanujan [8] has found what is equivalent to a Turán expression for $P(\zeta)$, and we may state his result in the form of the

THEOREM 3 (Ramanujan). Let $x>1, x+a>1, x+b>1$, $x+a+b>1$. Then

$$
\begin{aligned}
P(\zeta) & =\zeta(x) \zeta(x+a) \zeta(x+b) \zeta(x+a+b) \\
= & \infty(2 x+a+b) \quad \sum_{n}^{\infty} \frac{a^{(n) \sigma} b(n)}{n^{x+a+b}} .
\end{aligned}
$$

This relation is also given in Hardy and Wright [6, p. 256], as well as in many standard references on the Zeta function.

We may readily establish a formula for $T(1 / 5)$ by recalling a formula for the reciprocal of the Zeta function, 
using the Móbius function $\mu(n)$. We have

$$
\frac{1}{\zeta(x)}=\sum_{n=1}^{\infty} \frac{\stackrel{(L)}{\mu}(n)}{x}, x>1
$$

which yields

$$
\frac{1}{\zeta(x+a) \zeta(x+b)}=\sum_{n=1}^{\infty} \frac{1}{n^{x+a}} \sum_{d \mid n} d^{a-b} \mu(d) \mu(n / d)
$$

and we then find

$$
T(1 / \zeta)=\sum_{n=1}^{\infty} \frac{1}{n+a+b} \sum_{d \mid n} \mu(d) \mu(n / d) d^{a}\left[\left(\frac{n}{d}\right)^{b}-d^{b}\right]
$$

Thus we find

THEOREM 4. Let $x>1, x+a>1, x+b>1, x+a+b>1$.

Then

$$
T(1 / \zeta)=-\sum_{n=1}^{\infty} \frac{1}{n^{x+a+b}} \sum_{\substack{d<\sqrt{n} \\ d<\sqrt{n}}} \mu(d) \mu\left(\frac{n}{d}\right)\left[\left(\frac{n}{d}\right)^{a}-d^{a}\right]\left[\left(\frac{n}{d}\right)-d b\right] .
$$

Ramanujan [8] remarked that he had found the various relations (among them the one equivalent to (2.15) above) incidentally in the course of his other investigations. He said that "none of them seem to be of particular importance, nor does their proof involve the use of any new ideas, but some of them are so curious that they seem to be worth printing. "It is in the same spirit that we offer the relations developed here. It is interesting to note that Ramanujan also gave an elegant formula for $P(\eta)$ where

$$
\eta(x)=\sum_{n=0}^{\infty} \frac{(-1)^{n}}{(2 n+1)^{x}}
$$

The relation is very similar to (2.15) and involves alternating 
signs. The Turán expressions for $\eta(x)$ could be developed in much the same way as we have found them for $\zeta(x)$.

\section{REFERENCES}

1. S.K. Chatterjea, On an associated function of Hermite polynomials, Math. Z., 78(1962), 116-121.

2. A.E. Danese, Explicit evaluations of Turán expressions, Ann. Mat. Pura Appl., 38(1955), 339-348.

3. H.W. Gould, A generalization of a problem of L. Lorch and L. Moser, Canad. Math. BuII., 4(1961), 303-305.

4. H.W. Gould, The operator $T f(x)=f(x+a) f(x+b)-f(x) f(x+a+b)$, to appear in the Math. Mag.

5. H.W. Gould, Notes on a calculus of Turán operators, Mathematica Monongaliae, No.6, May, 1962.

6. G. H. Hardy and E.M. Wright, An Introduction to the Theory of Numbers (3rd Ed.), Oxford, 1954.

7. N.E. Nörlund, Vorlesungen über Differenzenrechnung, New York, 1954.

8. S. Ramanujan, Some formulae in the analytic theory of numbers, Messenger of Math., 45(1916), 81-84.

9. G. Szegó, On an inequality of P. Turán concerning Legendre polynomials, Bull. Amer. Math. Soc., 54(1948), $401-405$.

10. Paul Turán, On the zeros of the polynomials of Legendre. Ćasopis pést. mat., 75(1950), 113-122.

11. B.M. Wilson, Proofs of some formulae enunciated by Ramanijan, Proc. London Math. Soc., (2)21(1923). $235-255$.

12. Problem E 1396, Amer. Math. Monthly, 67(1960), 81-82; solution, 67(1960), 694.

West Virginia University

Morgantown, W. Va. 